\title{
A study of the average effect of the 3'APOB-VNTR polymorphism on lipidemic parameters could explain why the short alleles $(<35$ repeats) are rare in centenarians
}

\author{
Sabrina Garasto ${ }^{1,2}$, Maurizio Berardelli1,2, Francesco DeRango1, \\ Vincenzo Mari², Emidio Feraco ${ }^{2}$ and Giovanna De Benedictis*1
}

Address: ${ }^{1}$ Department of Cell Biology, University of Calabria.87030 Rende, Italy and ${ }^{2}$ Italian National Research Center on Aging (INRCA) 87100 Cosenza, Italy

Email: Sabrina Garasto - sabrina_gar@yahoo.it; Maurizio Berardelli - mberardelli@libero.it; Francesco DeRango - fderango@unical.it; Vincenzo Mari - v.mari@inrca.it; Emidio Feraco - e.feraco@inrca.it; Giovanna De Benedictis* - g.debenedictis@unical.it

* Corresponding author

Published: 09 February 2004

BMC Medical Genetics 2004, 5:3

This article is available from: http://www.biomedcentral.com/I47I-2350/5/3

(c) 2004 Garasto et al; licensee BioMed Central Ltd. This is an Open Access article: verbatim copying and redistribution of this article are permitted in all media for any purpose, provided this notice is preserved along with the article's original URL.
Received: II October 2003

Accepted: 09 February 2004

\begin{abstract}
Background: In studies on the genetics of human aging, we observed an age-related variation of the 3'APOBVNTR genotypic pool (alleles: Short, S, <35 repeats; Medium, M, 35-39 repeats; Long, L, >39 repeats) with the homozygous SS genotype showing a convex frequency trajectory in a healthy aging population. This genotype was rare in centenarians, thus indicating that the $S$ alleles are unfavorable to longevity, while common in adults, thus indicating a protective role at middle age. This apparent paradox could be due to possible effects exerted by the above polymorphism on lipidemic parameters. Aim of the work was to get insights into these puzzling findings

Methods: We followed a double strategy. Firstly, we analyzed the average effects of $S\left(\alpha_{S}\right), M\left(\alpha_{M}\right)$, and $L\left(\alpha_{L}\right)$ alleles on lipidemic parameters in a sample of healthy people (409 subjects aged 20-102 years) recruited in Calabria (southern Italy). The $\left(\alpha_{S}\right),\left(\alpha_{M}\right)$, and $\left(\alpha_{L}\right)$ values were estimated by relating 3'APOB-VNTR genotypes to lipidemic parameters, after adjustment for age, sex and body mass index (multiple regression). Then, we analyzed the $S$ alleles as susceptibility factors of Cardiovascular Atherosclerotic Disease (CD) in CD patients characterized either by low serum HDL-Cholesterol or by high serum LDL-Cholesterol (CD-H and CD-L patients, 40 and 40 subjects respectively). The Odds Ratios (OR) were computed for carriers of $S$ alleles in CD-H and CD-L patients matched for origin, sex and age with controls extracted from the sample of healthy subjects.

Results: By the analysis of the healthy sample group we found that the $S$ alleles lower the average values of serum Total Cholesterol $\left(\alpha_{\mathrm{S}}=-5.98 \mathrm{mg} / \mathrm{dL}\right.$ with [-I I.62 $\left.\div-0.74\right] 95 \%$ confidence interval) and LDL-Cholesterol $\left(\alpha_{\mathrm{S}}=-\right.$ $4.41 \mathrm{mg} / \mathrm{dL}$ with $[-8.93 \div-0.20] 95 \%$ confidence interval) while the alleles $M$ and $L$ have no significant effect on the lipidemic phenotype. In line with these findings, the analysis of CD patients showed that the $S$ alleles are protective as for CD-L (O.R. = 0.55 with $[0.21 \div 0.98] 95 \%$ confidence interval) while neutral as for CD-H $(O . R .=0.75$ with $[0.32 \div 1.60] 95 \%$ confidence interval).

Conclusion: On the whole, the $S$ alleles would be advantageous in adults (by protecting from CD-L) while dangerous in the elderly, probably by lowering serum cholesterol below a critical threshold. This could explain the convex frequency trajectory of SS genotypes previously observed in a healthy aging population.
\end{abstract}




\section{Background}

It is well known that the serum level of Low Density Lipoprotein - Cholesterol (LDL-C) is positively correlated with proneness to atherosclerosis and cardiovascular diseases. Apolipoprotein $\mathrm{B}(\mathrm{ApoB})$ is the main component of LDL and the variability of the APOB gene may play a role in the genetic susceptibility to such diseases by modulating the average effects exerted by APOB variants on the lipidemic phenotype $[1,2]$. A VNTR polymorphism, which is located $75 \mathrm{bp}$ downstream the second polyadenylation signal at the 3' end of the APOB gene (2p24-p23) has been suggested as one of the factors which modulate liability to coronary heart disease, at least in populations of Caucasian ancestry $[3,4]$ possibly through allele-specific expressions of the APOB gene [5].

Recently, in gene/longevity association studies, we analyzed the changes of the gene pool relevant to the 3'APOBVNTR polymorphism in healthy aging populations. By recoding the 3'APOB-VNTR alleles into three size classes (Small, S, <35 repeats; Medium, M, 35-39 repeats; Large, $L,>39$ repeats) we found that the frequency of the homozygous SS genotype was very low in oldest old subjects [6] and decreased in the population following an age-related convex trajectory [7]. The non linear trajectory suggested that the SS genotype was protective in younger individuals, while frail in the new scenario that comes out when age increases [8]. This puzzling finding could be due, at least in part, to a possible association between the 3'APOB-VNTR polymorphism and lipidemic parameters. Aim of the present study was to check this idea by analyzing the average effects of $S\left(\alpha_{S}\right), M\left(\alpha_{M}\right)$, and $L\left(\alpha_{L}\right)$ alleles on lipidemic parameters in a sample group of healthy subjects. Then, to check the indications given by the study of the allele average effect, we also analyzed patients affected by Cardiovascular Atherosclerotic Disease (CD) who were characterized either by low serum HDL-Cholesterol or by high serum LDL-Cholesterol (CD-H and CD-L patients respectively).

\section{Methods \\ Healthy group}

The sampling was carried out in a well defined geographic area (Calabria, southern Italy) taking care to minimize possible heterogeneity factors as previously described [9]. The samples were recruited by an appropriate campaign focused on University' students and staff (18- to 60-year age range), thermal baths and University for the Elderly (61- to 95-year age range); while the centenarians were identified by consulting the Population Registers of the Municipalities of Calabria, contacted by phone and then visited. Routine blood analyses were carried out in each subject and, in addition, subjects older than 60 years underwent a complete clinical and geriatric assessment. Subjects having blood parameters in the sex- and age-spe- cific normal range, none taking regular medication, and free of clinically overt pathologies were enrolled in the study (169 males and 240 females; 20-102 years agerange). In particular, centenarians were from the categories $\mathrm{A}$ and $\mathrm{B}$ previously described [10].

\section{Patients}

Eighty male patients affected by Cardiovascular Atherosclerotic Disease (46-80 years age-range) and characterized either by a low level of serum HDL-C (CD-H patients, $n=40$ ) or by a high level of serum LDL-C (CD-L patients, $\mathrm{n}=40$ ) were recruited at the Italian National Research Center on Aging (INRCA) in Cosenza (Calabria, southern Italy). About $70 \%$ of the patients were settled in the hospital, while the remaining were ambulatory visited. All the patients were matched for ethnicity, origin, residence, age and sex with the appropriate sub-group of healthy subjects. Diagnostic criteria for the inclusion in the study are reported in Table 1, while Table 2 shows lipidemic parameters in CD-H and CD-L patients.

All the subjects, both in the sample of healthy people and in that of patients, were unrelated and had given their written informed consent to the present study.

\section{Molecular analyses}

The 3'APOB-VNTR polymorphism was typed as described [6] on PCR-amplified DNA extracted from blood buffy coats.

As for the lipidemic parameters, serum Total Cholesterol (TC), HDL-Cholesterol (HDL-C), Tryglycerides (TG) were measured in each subject (12 hrs. overnight fast) by enzymatic colorimetric method with commercial kits (Dade Behring) in an automated chemistry analyzer (Dimension $^{\mathrm{R}}$ Clinical Chemistry System). LDL-Cholesterol (LDLC) was estimated by the Friedewald's formula [11].

\section{Statistical analyses}

The $\alpha_{S}, \alpha_{M}, \alpha_{L}$ average effects exerted by $S, M$, and $L$ alleles respectively on the lipidemic parameters were estimated in the sample of healthy subjects according to Christiansen and Feldman [12] after verification of the HardyWeinberg equilibrium ( $\chi^{2}$ test). Lipidemic data were used after removing trends due to age, sex and Body Mass Index (computed according to the formula: $\mathrm{BMI}=$ weight $[\mathrm{Kg}] /$ height $\left.\left[\mathrm{m}^{2}\right]\right)$ by multiple regression. The 95\% confidence intervals of $\alpha_{S}, \alpha_{M}, \alpha_{L}$ were calculated by bootstrap (1,000 replications, MATLAB Statistic toolbox).

The Odds Ratios (OR) in CD-H and CD-L were estimated for the carriers of $S$ alleles (homozygous plus heterozygous subjects) with respect to sex- and age-matched controls extracted from the sample of healthy subjects. 
Table I: Diagnostic criteria used to identify CD-H and CD-L patients with Cardiovascular Atherosclerotic Disease (CD)

Patients with Cardiovascular Atherosclerotic Disease (CD)

$\begin{aligned} & \text { Coronary Artery Disease } \\ & \text { - Myocardial Infarction }\end{aligned}$
- Angina Pectoris
- PTCA (Percutaneous Transfemoral
Coronary Angioplasty)
- CABG (Coronary Artery By-pass Graft)
$\begin{aligned} & \text { Carotid Atherosclerosis } \\ & \text { Peripheral Arterial Disease }\end{aligned}$
$\begin{aligned} & \text { HDL-Cholesterol } \\ & \text { LDL-Cholesterol } \\ & \text { With or Without } \\ & \text { Tryglycerides }\end{aligned}$

*Cutoff values were chosen on the basis of the average serum levels of HDL-C and LDL-C in southern Italian adult males not affected by any clinically manifest disease.

Table 2: Lipidemic parameters $(\mathrm{mg} / \mathrm{dL})$ in the samples of CD patients (46-80 years age range). Mean values with standard deviation in parenthesis.

\begin{tabular}{cc} 
CD-H Patients $(n=40 ;$ median age 64 years) & CD-L Patients $(n=40 ;$ median age 62 years) \\
\hline $185.7(44.7)$ & $284.6(34.1)$ \\
$27.8(3.2)$ & $45.4(9.9)$ \\
$125.6(32.1)$ & $207.3(30.6)$ \\
$2.15(0.2)$ & $2.2(0.2)$
\end{tabular}

CD-H: Cardiovascular Disease with low level of HDL-C; CD-L: Cardiovascular Disease with high level of LDL-C; TC: serum Total Cholesterol; HDL-C: serum HDL-Cholesterol; LDL-C: serum LDL-Cholesterol (TC - HDL-C - TG/5); TG: serum Tryglycerides

The 95\% confidence intervals of OR were computed by bootstrap (1,000 replications, MATLAB Statistic toolbox).

\section{Results}

\section{Estimation of allele-average effects}

The genotype/phenotype relationship between 3'APOBVNTR variability and lipidemic parameters was analyzed in the sample of healthy subjects by estimation of allelic average effects on lipidemic parameters. Tables 3 and 4 summarize lipidemic data (stratified according to age and sex) and genetic data respectively. The allele average effects $\alpha_{S^{\prime}} \alpha_{M^{\prime}} \alpha_{L}$ on serum TC, HDL-C, LDL-C and TG, after adjustment for age, sex and Body Mass Index, are shown in Table 5, where it can be seen that the $S$ alleles significantly lower the serum level of both TC and LDL-C (95\% c.i. non including 0).

\section{3'APOB-VNTR alleles in CD}

The genotypic and allelic frequencies in $\mathrm{CD}-\mathrm{H}$ and $\mathrm{CD}-\mathrm{L}$ patients, with those in sex- and age-matched controls used to compute OR values, are shown in the Table 6 . According with the results of the study of allele average effects, that showed a specific effect exerted by the $S$ alleles on both TC and LDL-C, we found that the $S$ alleles are protective in $\mathrm{CD}-\mathrm{L}(\mathrm{OR}=0.55 ;[0.21 \div 0.98] 95 \%$ c.i. $)$ while neutral in CD-H (O.R. $=0.75 ;[0.32 \div 1.60] 95 \%$ c.i. $)$.

\section{Discussion}

The study showed an allele-specific association between the 3 'APOB-VNTR variability and lipidemic parameters in a southern Italian population. Although association studies should be carefully considered due to the possibility of false positive findings, we are confident that the results of the present study are reliable for three reasons. Firstly, the 
Table 3: Lipidemic parameters $(\mathrm{mg} / \mathrm{dL})$ in the sample of healthy subjects stratified for age. Mean values with standard deviation in parenthesis.

\begin{tabular}{|c|c|c|c|}
\hline \multicolumn{4}{|c|}{ Males } \\
\hline Age & $20-45$ yrs. $n=54$ & $46-80$ yrs. $n=105$ & $\geq 100$ yrs. $n=10$ \\
\hline TC & $206.8(45.8)$ & $216.8(43.4)$ & $185.3(32.7)$ \\
\hline HDL-C & $48.9(9.9)$ & $57.1(16.5)$ & $47.0(14.0)$ \\
\hline LDL-C & $132.9(37.9)$ & I37.4 (37.3) & II $14.7(27.6)$ \\
\hline TG $\left(\log _{10}\right)$ & $2.0(0.2)$ & $2.1(0.2)$ & $2.0(0.1)$ \\
\hline \multicolumn{4}{|c|}{ Females } \\
\hline Age & $20-45$ yrs. $n=120$ & $46-80$ yrs. $n=80$ & $\geq 100$ yrs $n=40$ \\
\hline TC & $191.7(32.7)$ & $224.8(48.6)$ & I79.| (25.2) \\
\hline HDL-C & $63.7(13.2)$ & $65.3(15.4)$ & $50.2(13.7)$ \\
\hline LDL-C & $113.1(27.7)$ & $142.7(41.8)$ & $103.9(24.1)$ \\
\hline TG $\left(\log _{10}\right)$ & $1.8(0.2)$ & $2.0(0.2)$ & $2.0(0.2)$ \\
\hline
\end{tabular}

TC: serum Total Cholesterol; HDL-C: serum HDL-Cholesterol; LDL-C: serum LDL-Cholesterol (TC - HDL.C - TG/5); TG: serum Tryglycerides

Table 4: 3'APOB-VNTR genotypes, with Hardy-Weinberg equilibrium (HWE) expected values, in the sample of healthy subjects. Absolute and relative allelic frequencies (with standard errors) are also shown.

\begin{tabular}{|c|c|c|}
\hline Genotypes $(n=409)$ & Counts & *HWE \\
\hline SS & 15 & 17.05 \\
\hline$S M$ & 119 & 108.81 \\
\hline$S L$ & 18 & 24.09 \\
\hline$M M$ & 166 & 173.65 \\
\hline$M L$ & 82 & 76.89 \\
\hline$L L$ & 9 & 8.51 \\
\hline Alleles $(n=8 \mid 8)$ & Absolute & Relative \pm SE \\
\hline$s$ & 167 & $0.204 \pm 0.014$ \\
\hline$M$ & 533 & $0.652 \pm 0.017$ \\
\hline$L$ & 118 & $0.144 \pm 0.012$ \\
\hline
\end{tabular}

${ }^{*} \chi^{2}=3.44$ with 3 d.f.; $p=0.328$ Alleles: $S<35$ repeats; $M$ 35-39 repeats; $L>39$ repeats.

lowering effect exerted by the $3 `$ APOB-VNTR short alleles on serum TC and LDL-C had been previously observed in a sample of healthy people from Belgrade [13]. Secondly, the typing of a moderate number of neutral markers spread across the genome [14] did not show stratification in the sample we analyzed (unpublished results). Thirdly, and mostly important, the significant lowering effect exerted by the $S$ alleles on serum LDL-C (Table 5 ) is in line with the finding that these alleles are protective for CD-L (O.R. $=0.55$ with $[0.21 \div 0.98] 95 \%$ confidence interval) while neutral for CD-H.

The average effect exerted by the $S$ alleles on TC and LDL$\mathrm{C}$, and their protective role in $\mathrm{CD}-\mathrm{L}$, could explain why the
SS homozygous genotype was over-represented in an adult age-class of people selected for healthy status [7]. However, as age increases, both TC and LDL-C serum levels decline as shown by the population data in Table 3 . In this new physiological situation, the SS genotypes, that was protective at adult age, may turn out to be detrimental at old age, because the serum levels of TC and LDL-C may drop out below a critical threshold. In fact, in very old people, a high serum TC concentration is associated with increased survival $[15,16]$.

\section{Conclusions}

The data here presented showed that the $S$ alleles of the 3'APOB-VNTR polymorphism (less than 35 repeats) are 
Table 5: Allele average effects $\alpha_{S}, \alpha_{M}$ and $\alpha_{L}$ estimated for $S, \boldsymbol{M}$ and $L$ alleles respectively of the 3'VNTR-APOB polymorphism in the whole sample of healthy subjects after normalization for age, sex and BMI (computed according to the formula: BMI = weight [Kg]/ height $\left.\left[\mathrm{m}^{2}\right]\right)$

\begin{tabular}{ccccccc}
\hline & $\alpha_{\mathbf{S}}$ & $\mathbf{9 5 \%}$ c.i. $\alpha_{\mathbf{S}}$ & $\alpha_{\mathbf{M}}$ & $\mathbf{9 5 \%}$ c.i. $\alpha_{\mathbf{M}}$ & $\alpha_{\mathbf{L}}$ & $\mathbf{9 5 \%}$ c.i. $\alpha_{\mathbf{L}}$ \\
\hline TC & -5.98 & $-11.62 \div-0.74$ & +1.67 & $-0.28 \div+3.90$ & +0.93 & $-6.20 \div+8.13$ \\
HDL-C & -0.09 & $-2.01 \div+1.71$ & -0.08 & $-0.64 \div+0.81$ & +0.26 & $-2.51 \div+2.30$ \\
LDL-C & -4.41 & $-8.93 \div-0.20$ & +1.28 & $-0.65 \div+3.10$ & +0.45 & $-5.70 \div+6.82$ \\
TG $\left(\log _{10}\right)$ & +0.01 & $-0.02 \div+0.03$ & 0.00 & $-0.01 \div+0.01$ & -0.01 & $-0.04 \div+0.02$ \\
\hline
\end{tabular}

TC: serum Total Cholesterol; HDL-C: serum HDL-Cholesterol; LDL-C: serum LDL-Cholesterol; TG: serum Tryglycerides. Average effects are expressed in $\mathrm{mg} / \mathrm{dL}$. Confidence intervals (c.i.) are computed by bootstrap ( 1,000 replications).

Table 6: 3'APOB-VNTR absolute (Abs.) and Relative (Rel.) genotypic and allelic frequencies ( \pm Standard Error) in CD-H and CD-L patients and in sex and age matched controls (males; 46-80 year age range). Values expected at Hardy-Weinberg equilibrium (HWE) are in parenthesis. Alleles: $S<35$ repeats; $M$ 35-39 repeats; $L>39$ repeats.

\begin{tabular}{lllllll}
\hline Genotype & \multicolumn{2}{c}{ CD-H patients $(\mathbf{n}=\mathbf{4 0})$} & \multicolumn{2}{c}{ CD-L patients (n= 40) } & \multicolumn{2}{c}{ Controls (n= I05) } \\
Rel. \pm S.E.
\end{tabular}

associated with low levels of both TC and LDL-C in a population from southern Italy. This finding can explain why these alleles were over-represented in a sample of healthy adults [7] while rare in a sample of CD-L patients, characterized by high level of serum LDL-C. From this point of view, the $S$ alleles could be regarded as beneficial for healthy ageing. However, the observation that these alleles are rare in centenarians $[6,7]$ indicates that they are detrimental for longevity, probably because of their effect on lipidemic parameters in the physiological background of very old persons. On the whole, the study confirms that genetic risk factors are age-specific and gets possible insights on another "paradox of centenarians" [17].

\section{Competing interests}

None declared.

\section{Authors' contributions}

SG: genotyping; MB: sampling and clinical analyses; FDR: data mining; VM: laboratory and clinical analyses; EF clin- ical diagnosis; GDB: work hypothesis, study design, and coordination. All the authors discussed the data and participated to the draft of the manuscript, that was finalized by GDB.

\section{Acknowledgements}

Work funded by the Italian Ministry of Health (IRCCS 2000-2002. Marcatori biologici di invecchiamento normale e patologico) and by the Italian National Research Center on Aging (INRCA, Ancona and Cosenza, Italy).

\section{References}

I. Young SG: Recent progress in understanding apolipoprotein B. Circulation 1990, 82: 1574-94.

2. Hallman DM, Visvikis S, Steinmetz J et al:: The effect of variation in the apolipoprotein B gene on plasmid lipid and apolipoprotein B levels. A likelihood-based approach to cladistic analysis. Ann Hum Genet 1994, 58:35-64.

3. Hegele RA, Huang LS, Herbert PN et al.: Apolipoprotein B-gene DNA polymorphisms associated with myocardial infarction. N Engl J Med 1986, 315:1509-15.

4. Friedl W, Ludwig EH, Paulweber B et al:: Hypervariability in a minisatellite $3^{\prime}$ of the apolipoprotein $B$ gene in patients with coronary heart disease compared with normal controls. J Lipid Res 1990, 31:659-65. 
5. Chen B, Guo Z, He $P$ et al:: Structure and function of alleles in the $3^{\prime}$ end region of human apoB gene. Chin Med J 1999, I I 2:22I-3.

6. De Benedictis G, Falcone E, Rose G et al.: DNA multiallelic systems reveal gene/longevity associations not detected by diallelic systems. The APOB locus. Hum Genet 1997, 99:3। 2-8.

7. De Benedictis G, Carotenuto L, Carrieri G et al.: Age-related changes of the 3'APOB-VNTR genotype pool in ageing cohorts. Ann Hum Genet 1998, 62: I 15-22.

8. Snieder H, van Doornen LJP, Boomsma DI: The age dependency of gene expression for plasma lipids, lipoproteins, and apolipoproteins. Am J Hum Genet 1997, 60:638-50.

9. Garasto S, Rose G, De Rango F et al.: The study of APOAI, APOC3 and APOA4 variability in healthy ageing people reveals another paradox in the oldest old subjects. Ann Hum Genet 2003, 67:54-62.

10. Franceschi C, Motta L, Valensin S, and the Italian Multicenter Study on Centenarians: Do men and women follow different trajectories to reach extreme longevity ? Aging Clin Exp Res 2000, I 2:77-84.

II. Friedewald WT, Levy RI, Fredrickson DS: Estimation of the concentration of low-density lipoprotein cholesterol in plasma, without use of the preparative ultracentrifuge. Clin Chem I972, 18:499-502.

12. Christiansen FB, Feldman MW: Population Genetics Balckwell Sc Publ; 1986:130-134.

13. Alavantic $D$, Glisic $S$, Kandic I: APO B 3' HVR polymorphism in healthy population: relationships to serum lipid levels. Genet Epidemiol 1998, 15: I 13-22.

14. Pritchard JK, Donnelly P: Case-control studies of association in structured or admixed populations. Theor Popul Biol 200I, 60:227-37.

15. Schatz IJ, Masaki K, Yano K et al.: Cholesterol and all-cause mortality in elderly people from the Honolulu Heart Program: a cohort study. Lancet 200I, 358:35I-5.

16. Weverling-Rijnburger AWE, Blauw GJ, Lagaay AM et al.: Total cholesterol and risk of mortality in the oldest old. Lancet 1997, 350: I I 19-23. Erratum in: Lancet 1998, 35: 70

17. Forette $B$ : Are common risk factors relevant in the eldest old ? In The Paradoxes of Longevity Edited by: Robine JM, Forette B, Franceschi C, Allard M. Berlin, Springer Verlag; 1999:73-80.

\section{Pre-publication history}

The pre-publication history for this paper can be accessed here:

http://www.biomedcentral.com/1471-2350/5/3/prepub
Publish with Bio Med Central and every scientist can read your work free of charge

"BioMed Central will be the most significant development for disseminating the results of biomedical research in our lifetime. "

Sir Paul Nurse, Cancer Research UK

Your research papers will be:

- available free of charge to the entire biomedical community

- peer reviewed and published immediately upon acceptance

- cited in PubMed and archived on PubMed Central

- yours - you keep the copyright
BioMedcentral 